\title{
The reduction in influenza rates in the United States during the COVID-19 pandemic
}

\author{
Anna Sabu-Kurian BS, Kripa Shrestha MBBS, MPH, MS, Sharmila Dissanaike MD, FACS, FCCM
}

\begin{abstract}
Influenza affects many lives worldwide each year during the months of October through February. The 2020-2021 influenza season saw a sharp decline in the cases reported in the United States and in other countries like Great Britain in comparison to previous influenza seasons. The most likely explanations for this decline are the safety measures taken during the COVID-19 pandemic, such as physical distancing, face mask use, and better hand hygiene in the mass population, which likely reduced the transmission and infection rates of influenza this season.
\end{abstract}

Keywords: influenza, surveillance, pandemic, transmission

\section{INTRODUCTION}

The COVID-19 pandemic in the United States has resulted in more than 346,000 deaths and 20 million infections in the year $2020 .{ }^{1}$ Standard public health recommendations during the pandemic include wearing masks or face-coverings, rigorous hand hygiene, avoiding crowds especially indoors, and maintaining at least 6 feet of physical distance. These measures, while not always followed, should theoretically reduce the spread of other droplet and airborne diseases, such as influenza. We therefore hypothesized that the influenza case rate would be lower in 2020-2021 compared to 2019-2020, as a byproduct of preventive measures adopted during the COVID-19 pandemic.

\section{Methods}

The influenza surveillance report database by the Centers for Disease Control and Prevention (CDC) was

Corresponding author: Sharmila Dissanaike Contact Information: Sharmila.Dissanaike@ttuhsc.edu DOI: 10.12746/swrccc.v9i39.839 queried for reported influenza tests and results in clinical laboratories. The CDC uses more than 300 clinical laboratories located in all 50 states, Puerto Rico, Guam, and the District of Columbia through the U.S. World Health Organization (WHO) Collaborating Laboratories System and the National Respiratory and Enteric Virus Surveillance System for virologic surveillance.

Influenza data from 2019-2020 season (September 29, 2019-January 4, 2020) were compared with the 2020-2021 season (September 27, 2020-January 2, 2021) based on positive cases of influenza subtypes $A$ and $B$ and the total number of tests performed in the United States.

\section{RESULTS}

Atotal of 517,839 clinical influenza tests with 70,218 positive cases were reported between September 29, 2019, and January 4, 2020; 397,934 total tests with 925 positive cases were reported between September 27, 2020, and January 2, 2021. The percentage of weekly positive influenza tests showed a significant decline in the 2020-2021 influenza season compared to the 2019-2020 influenza season (Figures 1 and 2).

The weekly mean influenza test positivity rate in the 2020-2021 influenza season $(0.0024 ; 95 \% \mathrm{Cl}$ : 


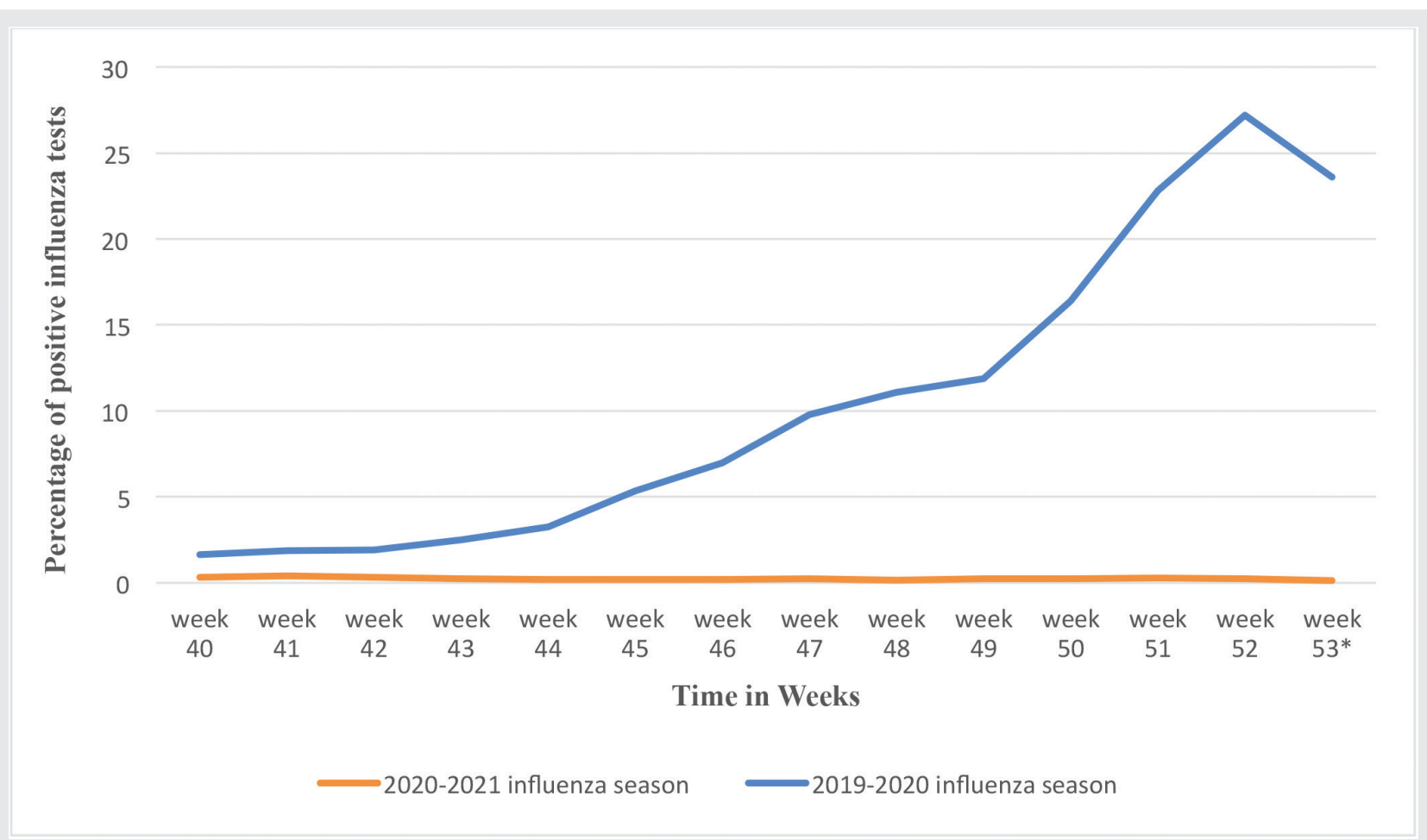

Figure 1. The percentage of positive influenza tests on a weekly basis. The blue line plot represents the 20192020 influenza season, which is reported from week 40, 2019 (September 29, 2019) to week 1, 2020 (January 4, 2020). The orange line plot represents the 2020-2021 influenza season, which is reported from week 40, 2020 (September 27, 2020) to week 53, 2020 (January 2, 2021).

* Week 53 in the 2020-2021 influenza season corresponds to Week 1 in the 2019-2020 influenza season.

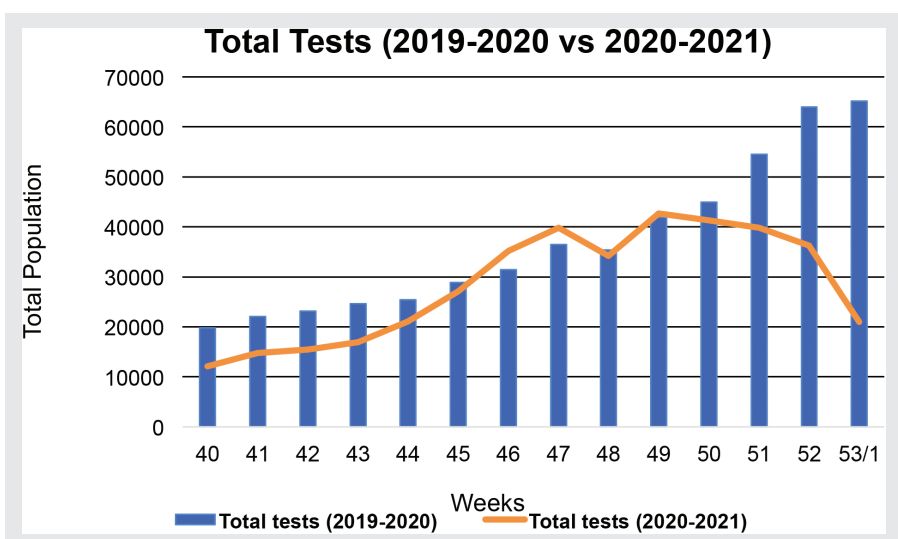

Figure 2. A total 517,839 clinical influenza tests were reported between September 29, 2019-January 4, 2020; whereas 397,934 total tests were reported between September 27, 2020-January 2, 2021.
0.0021-0.0028) was lower than the 2019-2020 influenza season rate $(0.10 ; 95 \% \mathrm{Cl}$ : $0.05-0.15)$. The weekly test positivity rate ratio was $<1$ on all weeks of comparison, indicating a decreased risk of influenza in the 2020-2021 season compared to 2019-2020.

\section{Discussion}

There was a marked decline in influenza cases in the United States last year. The most likely explanation for this decline involves measures taken across the nation to reduce the spread of COVID-19. Wearing masks/respirators can help reduce influenza transmission rates. ${ }^{2}$ Hand hygiene when combined with the use of facemasks has also shown efficacy against influenza infection. ${ }^{3}$ Reduction in travel and social events reduces the opportunity for the spread of all 
contagious diseases, especially those that are droplet or airborne.

Other contributing causes may be an increase in influenza vaccination rates and/or increased efficacy of the 2020 influenza vaccine. According to the CDC, 194-198 million influenza vaccine doses are projected for the 2020-2021 season, which is more than the 175 million doses given in the 2019-2020 season. ${ }^{4}$ A reduction in overall testing may have contributed to this substantial drop in rates, but since testing is predicated on symptoms, and there is no evidence of a decline in access to testing across the country, this is unlikely to be the primary factor in the decline. It is also possible that co-infections of influenza and COVID-19 were primarily reported as COVID rather than influenza, resulting in an artificial decrease in numbers, but this seems unlikely to account for the magnitude of decline.

Even with considerable testing, the average reported influenza cases in southern hemisphere countries like Australia were significantly lower with about 7,029 cases (March-September), while the average for similar periods in previous years has been $149,832 .{ }^{5}$ In the Great Britain during the 2019-2020 influenza season, the highest percentage of weekly positive influenza tests was $104.43 \%$ (week 1,2020 ), while it was $0.56 \%$ (week 1,2021 ) during the 2020-2021 influenza season. Many countries, e.g., China, Russia, and Argentina, have similar trends, suggesting that the decline in influenza was a worldwide phenomenon, relatively unaffected by geography. ${ }^{6}$

In conclusion, there has been a significant decrease in influenza in 2020-2021, most likely as a result of preventive measures adopted for the COVID-19 pandemic. This suggests that public health would benefit if similar precautions-hand hygiene, face covering, and physical distance-were adopted during future influenza seasons, even after the pandemic has subsided.
Article citation: Sabu-Kurian A, Shrestha K, Dissanaike S. The reduction in influenza rates in the United States during the COVID-19 pandemic. The Southwest Respiratory and Critical Care Chronicles 2021;9(39):22-24 From: Department of Surgery, Texas Tech University Health Sciences Center, Lubbock, Texas

Submitted: $1 / 29 / 2021$

Accepted: 2/21/2021

Reviewer: Gilbert Berdine MD

Conflicts of interest: none

This work is licensed under a Creative Commons Attribution-Share A like 4.0 International License.

\section{REFERENCES}

1. A Timeline of COVID-19 Developments in 2020. AJMC. https://www.ajmc.com/view/a-timeline-of-covid19-developments-in-2020. Accessed January 14, 2021.

2. Cowling BJ, Zhou Y, Ip DK, et al. Face masks to prevent transmission of influenza virus: a systematic review. Epidemiol Infect. 2010;138(4):449-456. doi:10.1017/S0950268809991658

3. Wong VW, Cowling BJ, Aiello AE. Hand hygiene and risk of influenza virus infections in the community: a systematic review and meta-analysis. Epidemiol Infect. 2014;142(5): 922-932.

4. Frequently Asked Influenza (Flu) Questions: 2020-2021 Season. Centers for Disease Control and Prevention. https://www. cdc.gov/flu/season/faq-flu-season-2020-2021.htm\#: :text= Flu\%20vaccine $\% 20$ is \%20produced\%20by,the $\% 202019 \%$ 2D2020\%20flu\%20season. Accessed January 14, 2021.

5. Sullivan SG, Carlson S, Cheng AC, et al. Where has all the influenza gone? The impact of COVID-19 on the circulation of influenza and other respiratory viruses, Australia, March to September 2020. Euro Surveill. 2020;25(47):2001847. doi: 10.2807/1560-7917.ES.2020.25.47.2001847

6. Power BI Report [Internet]. Powerbi.com. [cited $2021 \mathrm{Feb}$ 5]. Available from: https://app.powerbi.com/view?r=eyJrljoiYWU4YjUyN2YtMDBkOC00MGI1LTlhN2UtZGE5NThjY2E1ZThhIiwidCI6ImY2MTBjMGI3LWJkMjQtNGIzOS04MTBiLTNkYzI4MGFmYjU5MCIsImMiOjh9 